\title{
Unique solvability and stability of the time domain electromagnetic scattering problem with a locally perturbed perfectly conducting plate
}

\author{
Minfu Zhang ${ }^{1}$, Fuming $\mathrm{Ma}^{1}$ and Bo Chen ${ }^{2 *}$ (B)
}

"Correspondence:

charliecb@163.com

${ }^{2}$ College of Science, Civil Aviation

University of China, Tianjin, China

Full list of author information is

available at the end of the article

\begin{abstract}
In this paper, we investigate the unique solvability and stability of the time domain electromagnetic scattering problem with a kind of unbounded scatterer, that is, a locally perturbed perfectly electrical conducting plate. Specific analysis is provided for the perfectly electrical conducting boundary condition and Maxwell's equations to accomplish the symmetric continuation, and a symmetric scattering problem with a bounded scatterer is obtained. To analyze the unique solvability and stability of time domain electromagnetic scattering problems, Fourier-Laplace transformation and a "Laplace domain to time domain" analysis are involved. A rigorous analysis implies the unique solvability and stability of the scattering problem with a locally perturbed plate and implies that the problem is equivalent to the symmetric scattering problem with a bounded scatterer.
\end{abstract}

MSC: 35Q61; 35A15; 35B35

Keywords: Maxwell's equations; Time domain; Local perturbation; Symmetric continuation; Unique existence; Stability

\section{Introduction}

Scattering problems of acoustic and electromagnetic waves have been intensively investigated in the past decades since they depend on rigorous mathematical analysis and are closely associated with application areas such as medical imaging, nondestructive testing, and geophysical exploration. The propagations of acoustic waves and electromagnetic waves enable the construction of a wave equation and Maxwell's equations, respectively, which play important roles in the category of wave propagation problems [1]. The corresponding scattering problems are referred to as time domain problems since both the equations involve a time variable, which hinders analysis of the problems. Analysis of time domain electromagnetic scattering problems is more difficult due to the vector form of Maxwell's equations. As a result, researchers devote more time and energy to a simpler case, that is, time harmonic problems. In the time harmonic case, using tools such as Fourier transformation and polarization, analyses of the wave equation and Maxwell's equations are simplified as analyses of the Helmholtz equation $[2,3]$ or the time harmonic

(c) The Author(s) 2019. This article is distributed under the terms of the Creative Commons Attribution 4.0 International License (http://creativecommons.org/licenses/by/4.0/), which permits unrestricted use, distribution, and reproduction in any medium, provided you give appropriate credit to the original author(s) and the source, provide a link to the Creative Commons license, and indicate if changes were made. 
Maxwell systems $[4,5]$, which are independent of the time variable and the corresponding scattering problem is usually referred to as a frequency domain problem. A variety of frequency domain scattering problems with unbounded scatterers, such as locally perturbed half-planes [6-9] and open cavities [10-12], have been intensively explored. Newton type methods and sampling methods are considered in [6] and [7], respectively, to solve time harmonic electromagnetic scattering problems in locally perturbed half-planes. Mixed reciprocity relation and factorization method are applied to deal with the Helmholtz equation in [8], and an analysis of time harmonic Maxwell systems is provided in [9]. Time harmonic electromagnetic scattering problems with a cavity in a ground plane are considered in [10], in which a transparent boundary condition is proposed to solve the problem. Stability of time harmonic electromagnetic scattering from two-dimensional large open cavities is investigated in [11]. Integral formulation for electromagnetic scattering from three-dimensional large cavities is provided in [12].

Although the scattering problem with a time variable can be directly analyzed [13, 14], most time domain analyses are related to the frequency domain research on some level. In recent years, with the development of frequency domain research and computer techniques, the analysis and computation of time domain scattering problems have become possible and increasingly attracted the attention of researchers. Generally speaking, most time domain scattering problems can be classified as initial boundary value problems, which are extensively studied in various fields of mathematical physics [15].

For the acoustic case, via a Fourier-Laplace transformation of a time domain problem, a new problem that is sometimes referred to as the Laplace domain problem is obtained. The well-posedness, that is, the unique solvability and stability of time domain scattering problems, can be proven based on the Laplace domain analysis and a Fourier argument $[16,17]$. The analysis of [16] mainly depends on the layer potentials, which are important tools to deal with partial differential equations [18]. A detailed description of the theoretical basis of time domain acoustic scattering is provided in [19]. Well-posedness and time domain linear sampling methods to solve time domain scattering problems, such as the scattering by locally perturbed half-planes [20], Robin and Neumann obstacles [21], Dirichlet obstacles [22], and penetrable media [23], have been investigated. Kinds of time domain scattering problems with unbounded scatterers, such as periodic structures [24], rough surfaces [25], and locally perturbed half-planes [20], have also been analyzed.

For the time domain electromagnetic scattering problem, a similar "Laplace domain to time domain" analysis as the time domain acoustic scattering problem is involved to prove the well-posedness of the problem. Time domain electromagnetic scattering problems, such as the exterior scattering problem with a bounded scatterer [26], scattering by periodic structures [27] and rough surfaces [28], the open cavity problems [29-32], and scattering in chiral media [33], have been intensively analyzed. A brief survey of recent developments on analysis of the open cavity scattering problems is investigated in [29] for both the time harmonic case and the time domain case. The tools, such as the perfectly matched layer method [30], the transparent boundary condition [31], and the artificial boundary condition [32], have been investigated to truncate cavity scattering problems.

This paper concerns the analysis of time domain electromagnetic waves scattering by a locally perturbed perfectly conducting plate. Similar to the corresponding frequency domain works [6,9], we also make use of the symmetric continuation to solve the more 
complex time domain problem. The idea is inspired by the time domain acoustic scattering in a half-plane [20] and the Maxwell analysis in the time domain [26, 31].

For the convenience of the analysis and future computation, the scattering problem with a locally perturbed perfectly conducting plate is reformulated as a symmetric scattering problem with a bounded scatterer in this paper. In contrast to the Dirichlet boundary conditions of the frequency domain problem and the time domain acoustic scattering problem, a specific analysis is needed for the perfectly electrical conducting boundary condition and the Maxwell's equations to accomplish the symmetric continuation. For the reformulated problem, a Fourier-Laplace transform is employed to obtain a Laplace domain problem. The well-posedness of the Laplace domain problem is proved using the Lax-Milgram theorem. The well-posedness of the reformulated problem is determined by the "Laplace domain to time domain" analysis. The time domain electromagnetic scattering problem with a locally perturbed perfectly conducting plate is proved to be well posed and equivalent to the reformulated problem.

\section{Problem settings}

\subsection{Time domain electromagnetic scattering and a symmetric continuation}

Consider the scattering of time domain electromagnetic waves by a locally perturbed perfectly electrical conducting plate. Adopting Cartesian coordinates, denote by $\boldsymbol{x}=$ $\left(x_{1}, x_{2}, x_{3}\right)^{\mathrm{T}}$ a point in $\mathbb{R}^{3}$ and $\mathbb{R}_{0}^{3}=\left\{\boldsymbol{x} \in \mathbb{R}^{3}: x_{3}=0\right\}$. A sketch of the three-dimensional scattering problem is shown in Fig. 1. The upper boundary $\Gamma^{+}$of the local perturbation is assumed to be a smooth surface with its edge on $\mathbb{R}_{0}^{3}$. Denote $\Gamma^{c}=\mathbb{R}_{0}^{3} \backslash \Gamma_{0}$, where $\Gamma_{0} \subset \mathbb{R}_{0}^{3}$ is the lower surface of the local perturbation. The upper boundary of the locally perturbed plate is given by $\Gamma^{+} \cup \Gamma^{c}$, and the unbounded space upon the locally perturbed plate is denoted as $D_{e}^{+}$.

For $\boldsymbol{x} \in \mathbb{R}^{3}$ and $t \in \mathbb{R}$, the electromagnetic wave satisfies the time-dependent Maxwell's equations and the perfectly electrical conducting boundary condition

$$
\begin{aligned}
& \mu \frac{\partial \mathbf{H}}{\partial t}+\nabla \times \mathbf{E}=0 \quad \text { in } D_{e}^{+} \times \mathbb{R}, \\
& \varepsilon \frac{\partial \mathbf{E}}{\partial t}-\nabla \times \mathbf{H}=\mathbf{J} \quad \text { in } D_{e}^{+} \times \mathbb{R}, \\
& \mathbf{n} \times \mathbf{E}=0 \quad \text { on }\left\{\Gamma^{+} \cup \Gamma^{c}\right\} \times \mathbb{R},
\end{aligned}
$$

where $\varepsilon>0$ is the electric permittivity, $\mu>0$ is the magnetic permeability, $\mathbf{E}$ and $\mathbf{H}$ are the electric field intensity and the magnetic field intensity, respectively, and $\mathbf{J}$ is the current

Figure 1 Sketch of the three dimensional scattering problem

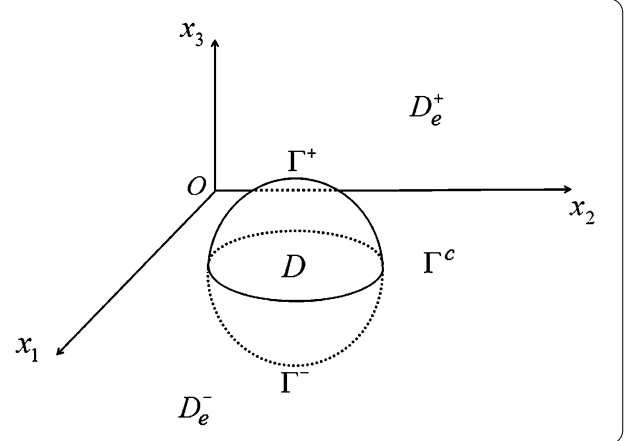


density with form

$$
\mathbf{J}(\boldsymbol{x}, t)=\rho(\boldsymbol{x}, t) \mathbf{v}(\boldsymbol{x}, t)
$$

where $\rho$ is the charge density and $\mathbf{v}$ is the particles' average drift velocity. In this paper, the charge density $\rho$ is assumed to be compactly supported in $D_{e}^{+}$, and the current density $\mathbf{J}$ is assumed to be causal, which indicates that $\mathbf{J}(\cdot, t)=0$ for $t<0$. The causality implies

$$
\mathbf{E}(\boldsymbol{x}, 0)=0, \quad \mathbf{H}(\boldsymbol{x}, 0)=0 .
$$

Due to the causality and compact support of the current density and the limited transmission speed of electromagnetic waves, the electromagnetic fields are compactly supported in space for any given $t$.

Denote

$$
T_{1} \mathbf{x}=\left(\begin{array}{ccc}
1 & 0 & 0 \\
0 & 1 & 0 \\
0 & 0 & -1
\end{array}\right) \boldsymbol{x}=\left(x_{1}, x_{2},-x_{3}\right)^{\mathrm{T}}
$$

as the symmetric point of $\boldsymbol{x} \in \mathbb{R}^{3}$ about the plane $\mathbb{R}_{0}^{3}$ and

$$
T_{2} \mathbf{x}=-T_{1} \mathbf{x}=\left(-x_{1},-x_{2}, x_{3}\right)^{\mathrm{T}} .
$$

Denote $\Gamma^{-}:=\left\{\boldsymbol{x}: T_{1} \boldsymbol{x} \in \Gamma^{+}\right\}$and $D_{e}^{-}:=\left\{\boldsymbol{x}: T_{1} \boldsymbol{x} \in D_{e}^{+}\right\}$as the reflection of $\Gamma^{+}$and the reflection of $D_{e}^{+}$, respectively. The symmetric continuation yields the bounded domain $D$ with the boundary $\partial D:=\Gamma^{+} \cup \Gamma^{-}$. The complementary set is denoted by $D_{e}=\mathbb{R}^{3} \backslash \bar{D}$.

Assume that

$$
\mathbf{J}(\boldsymbol{x}, t)=\left(\mathrm{J}_{1}(\boldsymbol{x}, t), \mathrm{J}_{2}(\boldsymbol{x}, t), \mathrm{J}_{3}(\boldsymbol{x}, t)\right)^{\mathrm{T}},
$$

and denote

$$
\mathbf{J}^{r}(\boldsymbol{x}, t)=T_{2} \mathbf{J}\left(T_{1} \mathbf{x}, t\right)=\left(-\mathrm{J}_{1}\left(T_{1} \boldsymbol{x}, t\right),-\mathrm{J}_{2}\left(T_{1} \mathbf{x}, t\right), \mathrm{J}_{3}\left(T_{1} \boldsymbol{x}, t\right)\right)^{\mathrm{T}}
$$

as the symmetric current density, which can be considered as a new current density with an opposite charge density compactly supported in $D_{e}^{-}$and a symmetric drift velocity, that is,

$$
\mathbf{J}^{r}(\boldsymbol{x}, t)=-\rho\left(T_{1} \boldsymbol{x}, t\right) T_{1} \mathbf{v}\left(T_{1} \boldsymbol{x}, t\right) .
$$

We consider a symmetric scattering problem with a bounded scatterer:

$$
\begin{aligned}
& \mu \frac{\partial \mathbf{H}}{\partial t}+\nabla \times \mathbf{E}=0 \quad \text { in } D_{e} \times \mathbb{R}, \\
& \varepsilon \frac{\partial \mathbf{E}}{\partial t}-\nabla \times \mathbf{H}=\mathbf{J}+\mathbf{J}^{r} \quad \text { in } D_{e} \times \mathbb{R}, \\
& \mathbf{n} \times \mathbf{E}=0 \quad \text { on } \partial D \times \mathbb{R} .
\end{aligned}
$$


The scattering problem (2.4)-(2.6) can be proved to be equivalent to problem (2.1)(2.3) after a well-posedness analysis. Before the analysis, we recall some notations about the spaces and the Fourier-Laplace transformation.

\subsection{Sobolev spaces and the Fourier-Laplace transform}

For the Lipschitz domain $D$, define the Hilbert spaces (refer to $[16,31,34]$ )

$$
\begin{aligned}
& \boldsymbol{H}(\operatorname{curl}, D)=\left\{\mathbf{U} \in \boldsymbol{L}^{2}(D), \nabla \times \mathbf{U} \in \boldsymbol{L}^{2}(D)\right\}, \\
& \boldsymbol{H}_{0}(\operatorname{curl}, D)=\{\mathbf{U} \in \boldsymbol{H}(\operatorname{curl}, D), \mathbf{n} \times \mathbf{U}=0 \text { on } \partial D\}
\end{aligned}
$$

with the same norm

$$
\|\mathbf{U}\|_{\boldsymbol{H}(\mathrm{curl}, D)}^{2}:=\|\mathbf{U}\|_{\mathbf{L}^{2}(D)}^{2}+\|\nabla \times \mathbf{U}\|_{\mathbf{L}^{2}(D)}^{2} .
$$

Set $\mathbb{C}_{\sigma}:=\{s \in \mathbb{C}: \operatorname{Re}(s) \geq \sigma\}$ for some $\sigma>0$ and $\mathbb{C}_{+}:=\{s \in \mathbb{C}: \operatorname{Re}(s)>0\}$. For $s \in \mathbb{C}_{\sigma}$, we recall the energy norm (refer to [16] Appendix A.3 for details)

$$
\|\mathbf{U}\|_{|s|, \Omega}^{2}:=\|s \mathbf{U}\|_{L^{2}(\Omega)}^{2}+\|\nabla \times \mathbf{U}\|_{L^{2}(\Omega)}^{2} .
$$

We recall some notations about the time-dependent Sobolev spaces (refer to [16, 20] for details). For a Hilbert space $\boldsymbol{X}$, denote the spaces of the $\boldsymbol{X}$-valued distributions and tempered distributions on the real line by $\mathcal{D}^{\prime}(\boldsymbol{X})$ and $\mathcal{S}^{\prime}(\boldsymbol{X})$, respectively.

The Fourier-Laplace transform and the corresponding inverse transform are defined as

$$
\check{\mathbf{U}}(s)=\mathcal{L}[\mathbf{U}](s):=\int_{-\infty}^{\infty} \mathrm{e}^{-s t} \mathbf{U}(t) \mathrm{d} t, \quad s \in \mathbb{C}_{\sigma}
$$

and

$$
\mathcal{L}^{-1}[\check{\mathbf{U}}](t):=\frac{1}{2 \pi \mathrm{i}} \int_{\sigma-\mathrm{i} \infty}^{\sigma+\mathrm{i} \infty} \mathrm{e}^{s t} \check{\mathbf{U}}(s) \mathrm{d} s
$$

respectively.

For $\sigma>0$, denote

$$
\mathcal{L}_{\sigma}^{\prime}(\mathbb{R}, \boldsymbol{X}):=\left\{\mathbf{U} \in \mathcal{D}^{\prime}(\boldsymbol{X}): \mathrm{e}^{-\sigma t} \mathbf{U} \in \mathcal{S}^{\prime}(\boldsymbol{X})\right\}
$$

and

$$
\mathcal{L}_{\sigma}^{\prime}\left(\mathbb{R}_{+}, \boldsymbol{X}\right):=\left\{\mathbf{U} \in \mathcal{L}_{\sigma}^{\prime}(\mathbb{R}, \boldsymbol{X}): \mathbf{U}(t)=0, \forall t<0\right\}
$$

For $\sigma>0$ and $p \in \mathbb{R}$, define the Sobolev space

$$
\boldsymbol{H}_{\sigma}^{p}(\mathbb{R}, \boldsymbol{X}):=\left\{\mathbf{U} \in \mathcal{L}_{\sigma}^{\prime}(\mathbb{R}, \boldsymbol{X}): \int_{\sigma-\mathrm{i} \infty}^{\sigma+\mathrm{i} \infty}|s|^{2 p}\|\mathcal{L}[\mathbf{U}](s)\|_{\boldsymbol{X}}^{2} \mathrm{~d} s<\infty\right\}
$$

with the norm

$$
\|\mathbf{U}\|_{\boldsymbol{H}_{\sigma}^{p}(\mathbb{R}, \boldsymbol{X})}:=\left(\int_{\sigma-\mathrm{i} \infty}^{\sigma+\mathrm{i} \infty}|s|^{2 p}\|\mathcal{L}[\mathbf{U}](s)\|_{\boldsymbol{X}}^{2} \mathrm{~d} s\right)^{1 / 2} .
$$




\section{Well-posedness}

\subsection{Well-posedness in the Laplace domain}

To show the well-posedness of the time domain scattering problems, firstly, the FourierLaplace transform is needed. The analysis is based on the analysis of the so-called Laplace domain problem.

Set $\check{\mathbf{E}}=\mathcal{L}\{\mathbf{E}\}, \check{\mathbf{H}}=\mathcal{L}\{\mathbf{H}\}, \check{\mathbf{J}}=\mathcal{L}\{\mathbf{J}\}$ and $\check{\mathbf{J}}^{r}=\mathcal{L}\left\{\mathbf{J}^{r}\right\}$. Recall that (refer to [16])

$$
\mathcal{L}\left\{\frac{\partial \mathbf{E}}{\partial t}\right\}=s \check{\mathbf{E}}, \quad \mathcal{L}\left\{\frac{\partial \mathbf{H}}{\partial t}\right\}=s \check{\mathbf{H}} .
$$

A Fourier-Laplace transform of equations (2.4)-(2.6) implies

$$
\begin{aligned}
& \mu s \check{\mathbf{H}}+\nabla \times \check{\mathbf{E}}=0 \quad \text { in } D_{e}, \\
& \varepsilon s \check{\mathbf{E}}-\nabla \times \check{\mathbf{H}}=\check{\mathbf{J}}+\check{\mathbf{J}}^{r} \quad \text { in } D_{e}, \\
& \mathbf{n} \times \check{\mathbf{E}}=0 \quad \text { on } \partial D,
\end{aligned}
$$

where $s \in \mathbb{C}_{+}$.

Equation (3.1) implies

$$
\check{\mathbf{H}}=-\frac{1}{\mu s} \nabla \times \check{\mathbf{E}} \quad \text { in } D_{e} .
$$

Submitting (3.4) into equation (3.2), consider the following auxiliary boundary value problem:

$$
\begin{aligned}
& \varepsilon s \check{\mathbf{E}}+\left(\nabla \times\left(\frac{1}{\mu s} \nabla \times \check{\mathbf{E}}\right)\right)=\check{\mathbf{J}}+\check{\mathbf{J}}^{r} \text { in } D_{e}, \\
& \mathbf{n} \times \check{\mathbf{E}}=0 \quad \text { on } \partial D .
\end{aligned}
$$

Proposition 3.1 Let $\breve{\mathbf{J}}$ be in the space $\mathbf{L}^{2}\left(D_{e}\right)$ and $s \in \mathbb{C}_{\sigma}$ for some $\sigma>0$. Then there exists a unique solution $\breve{\mathbf{E}} \in \boldsymbol{H}_{0}$ (curl, $D_{e}$ ) of problem (3.5)-(3.6). In addition,

$$
\|\check{\mathbf{E}}\|_{|s|, D_{e}} \leq C|s|\|\check{\mathbf{J}}\|_{L^{2}\left(D_{e}\right)},
$$

where $C$ is a constant that depends only on $\sigma, \mu$, and $\varepsilon$.

Proof Multiplying (3.5) by a test function $\mathbf{V} \in \boldsymbol{H}_{0}\left(\operatorname{curl}, D_{e}\right.$ ) and integrating in $D_{e}$, we obtain the variational formulation of problem (3.5)-(3.6): Find $\check{\mathbf{E}} \in \boldsymbol{H}_{0}$ (curl, $D_{e}$ ) such that

$$
a(\check{\mathbf{E}}, \mathbf{V})=F(\mathbf{V}), \quad \forall \mathbf{V} \in \boldsymbol{H}_{0}\left(\operatorname{curl}, D_{e}\right),
$$

where

$$
a(\check{\mathbf{E}}, \mathbf{V})=\frac{1}{\mu s}(\nabla \times(\nabla \times \check{\mathbf{E}}), \mathbf{V})_{L^{2}\left(D_{e}\right)}+\varepsilon s(\check{\mathbf{E}}, \mathbf{V})_{\mathbf{L}^{2}\left(D_{e}\right)}
$$

and

$$
F(\mathbf{V})=\left(\check{\mathbf{J}}+\check{\mathbf{J}}^{r}, \mathbf{V}\right)_{\mathbf{L}^{2}\left(D_{e}\right)} .
$$


For $\check{\mathbf{E}} \in \mathbf{H}_{0}\left(\right.$ curl, $\left.D_{e}\right)$, we obtain

$$
a(\check{\mathbf{E}}, \mathbf{V})=\frac{1}{\mu s}(\nabla \times \check{\mathbf{E}}, \nabla \times \mathbf{V})_{\mathbf{L}^{2}\left(D_{e}\right)}+\varepsilon s(\check{\mathbf{E}}, \mathbf{V})_{\mathbf{L}^{2}\left(D_{e}\right)}
$$

and

$$
\begin{aligned}
|a(\check{\mathbf{E}}, \mathbf{V})| & =\left|\frac{1}{\mu s}\left[(\nabla \times \check{\mathbf{E}}, \nabla \times \mathbf{V})_{L^{2}\left(D_{e}\right)}+\mu \varepsilon s^{2}(\check{\mathbf{E}}, \mathbf{V})_{L^{2}\left(D_{e}\right)}\right]\right| \\
& \leq \frac{2}{\mu|s|} \max \{1, \mu \varepsilon\}\|\check{\mathbf{E}}\|_{|s|, D_{e}}\|\mathbf{V}\|_{|s|, D_{e}} .
\end{aligned}
$$

Set $\mathbf{V}=\check{\mathbf{E}}$. Then we obtain

$$
\begin{aligned}
\operatorname{Re}\{a(\check{\mathbf{E}}, \check{\mathbf{E}})\} & =\operatorname{Re}\left\{\frac{\bar{s}}{\mu|s|^{2}}\|\nabla \times \check{\mathbf{E}}\|_{L^{2}\left(D_{e}\right)}^{2}+\frac{\varepsilon s}{|s|^{2}}|s|^{2}\|\check{\mathbf{E}}\|_{L^{2}\left(D_{e}\right)}^{2}\right\} \\
& =\frac{\operatorname{Re}\{s\}}{|s|^{2}}\left(\frac{1}{\mu}\|\nabla \times \check{\mathbf{E}}\|_{L^{2}\left(D_{e}\right)}^{2}+\varepsilon|s|^{2}\|\check{\mathbf{E}}\|_{L^{2}\left(D_{e}\right)}^{2}\right) \\
& \geq \sigma \min \left\{\frac{1}{\mu}, \varepsilon\right\}|s|^{-2}\|\check{\mid}\|_{|s|, D_{e}}^{2} .
\end{aligned}
$$

With the continuity of $F(\mathbf{V})$, according to the Lax-Milgram theorem, there exists a unique solution $\check{\mathbf{E}} \in \mathbf{H}_{0}(\mathrm{curl}, D)$ of problem (3.5)-(3.6).

Note that the symmetric property implies $\|\check{J}\|_{\mathbf{L}^{2}\left(D_{e}\right)}=\left\|\check{\boldsymbol{J}}^{r}\right\|_{\mathbf{L}^{2}\left(D_{e}\right)}$. The variational form and definition (2.7) imply that

$$
\begin{aligned}
|a(\check{\mathbf{E}}, \check{\mathbf{E}})| & =|F(\check{\mathbf{E}})|=\left(\check{\mathbf{J}}+\check{\mathbf{J}}^{r}, \check{\mathbf{E}}\right)_{\mathbf{L}^{2}\left(D_{e}\right)} \\
& \leq 2\|\check{\mathbf{J}}\|_{\mathbf{L}^{2}\left(D_{e}\right)}\|\check{\mathbf{E}}\|_{\mathbf{L}^{2}\left(D_{e}\right)} \\
& \leq 2|s|^{-1}\|\check{\mathbf{J}}\|_{\mathbf{L}^{2}\left(D_{e}\right)}\|\check{\mathbf{E}}\|_{|s|, D_{e}} .
\end{aligned}
$$

Thus

$$
\sigma \min \left\{\frac{1}{\mu}, \varepsilon\right\}|s|^{-2}\|\check{\mathbf{E}}\|_{|s|, D_{e}}^{2} \leq 2|s|^{-1}\|\check{\mathbf{J}}\|_{L^{2}\left(D_{e}\right)}\|\check{\mathbf{E}}\|_{|s|, D_{e}} .
$$

Then

$$
\|\check{\mathbf{E}}\|_{|s|, D_{e}} \leq C|s|\|\check{\mathbf{J}}\|_{L^{2}\left(D_{e}\right)},
$$

where $C$ is a constant that depends only on $\sigma, \mu$, and $\varepsilon$.

Proposition 3.2 Let $\breve{\mathbf{J}}$ be in the space $\mathbf{L}^{2}\left(D_{e}\right)$ and $s \in \mathbb{C}_{\sigma}$ for some $\sigma>0$. There exists a unique solution $(\check{\mathbf{E}}, \check{\mathbf{H}}) \in \boldsymbol{H}_{0}\left(\mathrm{curl}, D_{e}\right) \times \boldsymbol{H}\left(\mathrm{curl}, D_{e}\right)$ of problem (3.1)-(3.3). In addition,

$$
\|\check{\mathbf{E}}\|_{|s|, D_{e}} \leq C|s|\|\check{\mathbf{J}}\|_{L^{2}\left(D_{e}\right)}, \quad\|\check{\mathbf{H}}\|_{|s|, D_{e}} \leq C_{2}|s|\|\check{\mathbf{J}}\|_{L^{2}\left(D_{e}\right)}
$$

where $C$ and $C_{2}$ are constants that depend only on $\sigma, \mu$, and $\varepsilon$. 
Proof Equation (3.2) implies

$$
\nabla \times \check{\mathbf{H}}=\varepsilon s \check{\mathbf{E}}-\check{\mathbf{J}}-\check{\mathbf{J}}^{r} .
$$

Equations (3.4), (3.7) and Proposition 3.1 imply that there exists a unique solution $(\check{\mathbf{E}}, \check{\mathbf{H}}) \in$ $\mathbf{H}_{0}(\operatorname{curl}, D) \times \mathbf{H}(\operatorname{curl}, D)$ of problem (3.1)-(3.3) and

$$
\|\check{\mathbf{E}}\|_{|s|, D_{e}} \leq C|s|\|\check{\mathbf{J}}\|_{\mathbf{L}^{2}\left(D_{e}\right)},
$$

where $C$ is a constant that depends only on $\sigma, \mu$, and $\varepsilon$.

Definition (2.7) and equations (3.4) and (3.7) imply that

$$
\begin{aligned}
\|\check{\mathbf{H}}\|_{|s|, D_{e}}^{2}= & |s|^{2}\left\|-\frac{1}{\mu s} \nabla \times \check{\mathbf{E}}\right\|_{L^{2}\left(D_{e}\right)}^{2}+\left\|\varepsilon s \check{\mathbf{E}}-\check{\mathbf{J}}-\check{\mathbf{J}}^{r}\right\|_{L^{2}\left(D_{e}\right)}^{2} \\
\leq & \frac{1}{\mu^{2}}\|\nabla \times \check{\mathbf{E}}\|_{L^{2}\left(D_{e}\right)}^{2}+\varepsilon^{2}|s|^{2}\|\check{\mathbf{E}}\|_{L^{2}\left(D_{e}\right)}^{2}+4\|\check{\mathbf{J}}\|_{L^{2}\left(D_{e}\right)}^{2} \\
& +4 \varepsilon|s|\|\check{\mathbf{E}}\|_{L^{2}\left(D_{e}\right)}\|\check{\mathbf{J}}\|_{L^{2}\left(D_{e}\right)} \\
\leq & |s|^{2}\left(\max \left\{\frac{1}{\mu^{2}}, \varepsilon^{2}\right\} C^{2}+\frac{4}{\sigma^{2}}+\frac{4 \varepsilon C}{\sigma}\right)\|\check{\mathbf{J}}\|_{L^{2}\left(D_{e}\right)^{2}}^{2} .
\end{aligned}
$$

Denote

$$
C_{2}=\left(\max \left\{\frac{1}{\mu^{2}}, \varepsilon^{2}\right\} C^{2}+\frac{4}{\sigma^{2}}+\frac{4 \varepsilon C}{\sigma}\right)^{1 / 2} .
$$

The proposition is proved.

\subsection{Back to time domain}

Regarding the time domain, we obtain the following proposition about the scattering problem (2.4)-(2.6).

Proposition 3.3 Let $\sigma>0, p \in \mathbb{R}$, and $\mathbf{J} \in \boldsymbol{H}_{\sigma}^{p}\left(\mathbb{R}_{+}, \boldsymbol{L}^{2}\left(D_{e}\right)\right)$. There exists a unique solution $(\mathbf{E}, \mathbf{H}) \in \boldsymbol{H}_{\sigma}^{p-1}\left(\mathbb{R}_{+}, \boldsymbol{H}_{0}\left(\mathrm{curl}, D_{e}\right)\right) \times \boldsymbol{H}_{\sigma}^{p-1}\left(\mathbb{R}_{+}, \boldsymbol{H}\left(\mathrm{curl}, D_{e}\right)\right)$ of problem (2.4)-(2.6). In addition,

$$
\begin{aligned}
\|\mathbf{E}\|_{\boldsymbol{H}_{\sigma}^{p-1}\left(\mathbb{R}_{+}, \boldsymbol{H}_{0}\left(\operatorname{curl}, D_{e}\right)\right)} \leq C^{\prime}\|\mathbf{J}\|_{\boldsymbol{H}_{\sigma}^{p}\left(\mathbb{R}_{+}, \boldsymbol{H}\left(\operatorname{curl}, D_{e}\right)\right)}, \\
\|\mathbf{H}\|_{\boldsymbol{H}_{\sigma}^{p-1}\left(\mathbb{R}_{+}, \boldsymbol{H}\left(\operatorname{curl}, D_{e}\right)\right)} \leq C_{2}^{\prime}\|\mathbf{J}\|_{\boldsymbol{H}_{\sigma}^{p}\left(\mathbb{R}_{+}, \boldsymbol{H}\left(\operatorname{curl}, D_{e}\right)\right)},
\end{aligned}
$$

where $C^{\prime}$ and $C_{2}^{\prime}$ are constants that depend only on $\sigma, \mu$, and $\varepsilon$.

Proof According to Proposition 3.2, an application of Lemma 2 in [22] and a Fourier argument complete the proof.

$$
\text { For }(\boldsymbol{x}, t) \in D_{e} \times \mathbb{R} \text {, denote }
$$

$$
\mathbf{E}(\boldsymbol{x}, t)=\left(\mathrm{E}_{1}(\boldsymbol{x}, t), \mathrm{E}_{2}(\boldsymbol{x}, t), \mathrm{E}_{3}(\boldsymbol{x}, t)\right)^{\mathrm{T}}, \quad \mathbf{H}(\boldsymbol{x}, t)=\left(\mathrm{H}_{1}(\boldsymbol{x}, t), \mathrm{H}_{2}(\boldsymbol{x}, t), \mathrm{H}_{3}(\boldsymbol{x}, t)\right)^{\mathrm{T}}
$$

Notice that the scattering problem (2.4)-(2.6) has a symmetric structure, which is given by the following lemma. 
Lemma 3.4 Let $\sigma>0, p \in \mathbb{R}$, and $\mathbf{J} \in \boldsymbol{H}_{\sigma}^{p}\left(\mathbb{R}_{+}, \mathbf{L}^{2}\left(D_{e}\right)\right)$. Assume that $(\mathbf{E}, \mathbf{H}) \in \boldsymbol{H}_{\sigma}^{p-1}\left(\mathbb{R}_{+}\right.$, $\left.\boldsymbol{H}_{0}\left(\mathrm{curl}, D_{e}\right)\right) \times \boldsymbol{H}_{\sigma}^{p-1}\left(\mathbb{R}_{+}, \boldsymbol{H}\left(\mathrm{curl}, D_{e}\right)\right)$ is the unique solution of the scattering problem (2.4)(2.6). Then

$$
\mathbf{E}(\boldsymbol{x}, t)=T_{2} \mathbf{E}\left(T_{1} \boldsymbol{x}, t\right), \quad \mathbf{H}(\boldsymbol{x}, t)=T_{1} \mathbf{H}\left(T_{1} \boldsymbol{x}, t\right) .
$$

In addition,

$$
\mathbf{n} \times \mathbf{E}=0 \quad \text { on } \Gamma^{c} \times \mathbb{R} .
$$

Proof Firstly, we provide the analysis in the Laplace domain. Since $(\mathbf{E}, \mathbf{H})$ is the unique solution of the scattering problem (2.4)-(2.6), Proposition 3.1 implies that

$$
\begin{aligned}
\check{\mathbf{E}}(\boldsymbol{x} ; s) & =\left(\mathcal{L}\left\{\mathrm{E}_{1}\right\}(\boldsymbol{x} ; s), \mathcal{L}\left\{\mathrm{E}_{2}\right\}(\boldsymbol{x} ; s), \mathcal{L}\left\{\mathrm{E}_{3}\right\}(\boldsymbol{x} ; s)\right)^{\mathrm{T}} \\
& =\left(\check{\mathrm{E}}_{1}(\boldsymbol{x} ; s), \check{\mathrm{E}}_{2}(\boldsymbol{x} ; s), \check{\mathrm{E}}_{3}(\boldsymbol{x} ; s)\right)^{\mathrm{T}} \in \boldsymbol{H}_{0}\left(\operatorname{curl}, D_{e}\right)
\end{aligned}
$$

is the unique solution of problem (3.5)-(3.6).

For the linear system (3.5)-(3.6), the solution can be divided into $\check{\mathbf{E}}=\check{\mathbf{E}}_{1}+\check{\mathbf{E}}_{2}$, where $\check{\mathbf{E}}_{1}$ and $\check{\mathbf{E}}_{2}$ are the solutions of

$$
\begin{aligned}
& \varepsilon s \check{\mathbf{E}}+\left(\nabla \times\left(\frac{1}{\mu s} \nabla \times \check{\mathbf{E}}\right)\right)=\check{\mathbf{J}} \text { in } D_{e}, \\
& \mathbf{n} \times \check{\mathbf{E}}=0 \quad \text { on } \partial D
\end{aligned}
$$

and

$$
\begin{aligned}
& \varepsilon s \check{\mathbf{E}}+\left(\nabla \times\left(\frac{1}{\mu s} \nabla \times \check{\mathbf{E}}\right)\right)=\check{\mathbf{J}}^{r} \quad \text { in } D_{e}, \\
& \mathbf{n} \times \check{\mathbf{E}}=0 \quad \text { on } \partial D
\end{aligned}
$$

respectively. The Fourier-Laplace transformation implies

$$
\begin{aligned}
& \check{\mathbf{J}}(\boldsymbol{x} ; s)=\left(\check{\mathrm{J}}_{1}(\boldsymbol{x} ; s), \check{\mathrm{J}}_{2}(\boldsymbol{x} ; s), \check{\mathrm{J}}_{3}(\boldsymbol{x} ; s)\right)^{\mathrm{T}}, \\
& \check{\mathbf{J}}^{r}(\boldsymbol{x} ; s)=T_{2} \check{\mathbf{J}}\left(T_{1} \boldsymbol{x} ; s\right) .
\end{aligned}
$$

For $\check{\mathbf{E}}_{1}(\boldsymbol{x} ; s)=\left(\check{\mathrm{E}}_{11}(\boldsymbol{x} ; s), \check{\mathrm{E}}_{12}(\boldsymbol{x} ; s), \check{\mathrm{E}}_{13}(\boldsymbol{x} ; s)\right)^{\mathrm{T}}$, denote

$$
\check{\mathbf{E}}_{1}^{r}(\boldsymbol{x} ; s)=T_{2} \check{\mathbf{E}}_{1}\left(T_{1} \mathbf{x} ; s\right)
$$

Substituting $\check{\mathbf{E}}_{1}$ and $\check{\mathbf{J}}$ into (3.8) implies

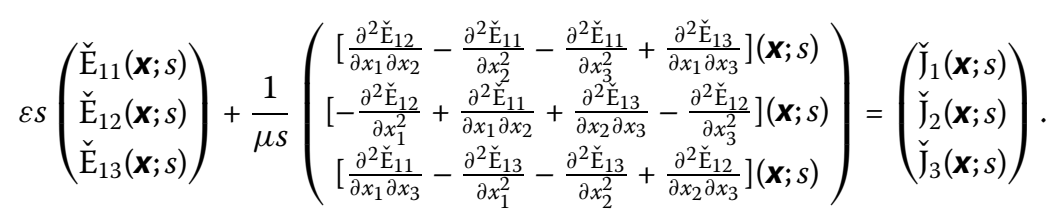


A direct computation implies that $\check{\mathbf{E}}_{1}^{r}(\boldsymbol{x} ; s)$ is also a solution of problem (3.10)-(3.11). For $\boldsymbol{x} \in D_{e}$, the unique solution $\check{\mathbf{E}}(\boldsymbol{x} ; s)$ can be represented as

$$
\begin{aligned}
\check{\mathbf{E}}(\boldsymbol{x} ; s) & =\check{\mathbf{E}}_{1}(\boldsymbol{x} ; s)+T_{2} \check{\mathbf{E}}_{1}\left(T_{1} \boldsymbol{x} ; s\right) \\
& =\left(\check{\mathrm{E}}_{11}(\boldsymbol{x} ; s)-\check{\mathrm{E}}_{11}\left(T_{1} \boldsymbol{x} ; s\right), \check{\mathrm{E}}_{12}(\boldsymbol{x} ; s)-\check{\mathrm{E}}_{12}\left(T_{1} \boldsymbol{x} ; s\right), \check{\mathrm{E}}_{13}(\boldsymbol{x} ; s)+\check{\mathrm{E}}_{13}\left(T_{1} \boldsymbol{x} ; s\right)\right)^{\mathrm{T}},
\end{aligned}
$$

which implies

$$
\check{\mathbf{E}}(\boldsymbol{x} ; s)=T_{2} \check{\mathbf{E}}\left(T_{1} \boldsymbol{x} ; s\right) \text {. }
$$

A Fourier argument implies that

$$
\mathbf{E}(\boldsymbol{x}, t)=T_{2} \mathbf{E}\left(T_{1} \mathbf{x}, t\right) .
$$

A direct computation based on equation (3.4) and a Fourier argument yield

$$
\mathbf{H}(\boldsymbol{x}, t)=T_{1} \mathbf{H}\left(T_{1} \boldsymbol{x}, t\right) .
$$

Note that $x_{3}=0$ on $\Gamma_{c}$, which implies $\boldsymbol{x}=T_{1} \boldsymbol{x}$. Thus

$$
\mathbf{n} \times \mathbf{E}=0 \quad \text { on } \Gamma^{c} \times \mathbb{R} .
$$

This completes the proof.

We present the following theorem to show the well-posedness of the scattering problem (2.1) $-(2.3)$ and the equivalence of the scattering problems (2.1)-(2.3) and (2.4)-(2.6).

Theorem 3.5 Let $\sigma>0, p \in \mathbb{R}$, and $\mathbf{J} \in \boldsymbol{H}_{\sigma}^{p}\left(\mathbb{R}_{+}, \mathbf{L}^{2}\left(D_{e}^{+}\right)\right)$. Then there exists a unique solution $\left(\mathbf{E}_{+}, \mathbf{H}_{+}\right) \in \boldsymbol{H}_{\sigma}^{p-1}\left(\mathbb{R}_{+}, \boldsymbol{H}_{0}\left(\mathrm{curl}, D_{e}^{+}\right)\right) \times \boldsymbol{H}_{\sigma}^{p-1}\left(\mathbb{R}_{+}, \boldsymbol{H}\left(\mathrm{curl}, D_{e}^{+}\right)\right)$of problem (2.1)-(2.3) which satisfies

$$
\begin{aligned}
\left\|\mathbf{E}_{+}\right\|_{\boldsymbol{H}_{\sigma}^{p-1}\left(\mathbb{R}_{+}, \boldsymbol{H}_{0}\left(\mathrm{curl}, D_{e}^{+}\right)\right)} \leq C^{\prime \prime}\|\mathbf{J}\|_{\boldsymbol{H}_{\sigma}^{p}\left(\mathbb{R}_{+}, \boldsymbol{H}\left(\mathrm{curl}, D_{e}^{+}\right)\right)}, \\
\left\|\mathbf{H}_{+}\right\|_{\boldsymbol{H}_{\sigma}^{p-1}\left(\mathbb{R}_{+}, \boldsymbol{H}\left(\mathrm{curl}, D_{e}^{+}\right)\right)} \leq C_{2}^{\prime \prime}\|\boldsymbol{J}\|_{\boldsymbol{H}_{\sigma}^{p}\left(\mathbb{R}_{+}, \boldsymbol{H}\left(\mathrm{curl}, D_{e}^{+}\right)\right)},
\end{aligned}
$$

where $C^{\prime \prime}$ and $C_{2}^{\prime \prime}$ are constants that depend only on $\sigma, \mu$, and $\varepsilon$. Moreover, if $(\mathbf{E}, \mathbf{H}) \in$ $\boldsymbol{H}_{\sigma}^{p-1}\left(\mathbb{R}_{+}, \boldsymbol{H}_{0}\left(\mathrm{curl}, D_{e}\right)\right) \times \boldsymbol{H}_{\sigma}^{p-1}\left(\mathbb{R}_{+}, \boldsymbol{H}\left(\mathrm{curl}, D_{e}\right)\right)$ is the unique solution of problem (2.4)-(2.6), we obtain

$$
\mathbf{E}_{+}=\left.\mathbf{E}\right|_{D_{e}^{+} \times \mathbb{R}}, \quad \mathbf{H}_{+}=\left.\mathbf{H}\right|_{D_{e}^{+} \times \mathbb{R}} .
$$

Proof Assume that $(\mathbf{E}, \mathbf{H})$ is the solution of problem (2.4)-(2.6). Denote

$$
\mathbf{E}_{+}=\left.\mathbf{E}\right|_{D_{e}^{+} \times \mathbb{R}}, \quad \mathbf{H}_{+}=\left.\mathbf{H}\right|_{D_{e}^{+} \times \mathbb{R}}
$$

According to Lemma 3.4,

$$
\left(\mathbf{E}_{+}, \mathbf{H}_{+}\right) \in \boldsymbol{H}_{\sigma}^{p-1}\left(\mathbb{R}_{+}, \boldsymbol{H}_{0}\left(\operatorname{curl}, D_{e}^{+}\right)\right) \times \boldsymbol{H}_{\sigma}^{p-1}\left(\mathbb{R}_{+}, \boldsymbol{H}\left(\operatorname{curl}, D_{e}^{+}\right)\right)
$$


is a solution of problem (2.1)-(2.3), which satisfies

$$
\begin{aligned}
\left\|\mathbf{E}_{+}\right\|_{\mathbf{H}_{\sigma}^{p-1}\left(\mathbb{R}_{+}, \mathbf{H}_{0}\left(\operatorname{curl}, D_{e}^{+}\right)\right)} \leq C^{\prime \prime}\|\mathbf{J}\|_{\mathbf{H}_{\sigma}^{p}\left(\mathbb{R}_{+}, \mathbf{H}\left(\operatorname{curl}, D_{e}^{+}\right)\right)}, \\
\left\|\mathbf{H}_{+}\right\|_{\mathbf{H}_{\sigma}^{p-1}\left(\mathbb{R}_{+}, \mathbf{H}\left(\operatorname{curl}, D_{e}^{+}\right)\right)} \leq C_{2}^{\prime \prime}\|\mathbf{J}\|_{\mathbf{H}_{\sigma}^{p}\left(\mathbb{R}_{+}, \mathbf{H}\left(\operatorname{curl}, D_{e}^{+}\right)\right)},
\end{aligned}
$$

where $C^{\prime \prime}$ and $C_{2}^{\prime \prime}$ are constants that depend only on $\sigma, \mu$, and $\varepsilon$.

We prove that $\left(\mathbf{E}_{+}, \mathbf{H}_{+}\right)$is a unique solution using reduction to absurdity. Assume that another solution

$$
\left(\mathbf{E}_{+}^{\prime}, \mathbf{H}_{+}^{\prime}\right) \in \boldsymbol{H}_{\sigma}^{p-1}\left(\mathbb{R}_{+}, \boldsymbol{H}_{0}\left(\operatorname{curl}, D_{e}^{+}\right)\right) \times \boldsymbol{H}_{\sigma}^{p-1}\left(\mathbb{R}_{+}, \boldsymbol{H}\left(\operatorname{curl}, D_{e}^{+}\right)\right)
$$

of problem (2.1)-(2.3) exists. Denote

$$
\mathbf{E}^{\prime}(\boldsymbol{x}, t)= \begin{cases}\mathbf{E}_{+}^{\prime}(\boldsymbol{x}, t), & (\boldsymbol{x}, t) \in D_{e}^{+} \times \mathbb{R} \\ T_{2} \mathbf{E}_{+}^{\prime}\left(T_{1} \mathbf{x}, t\right), & (\boldsymbol{x}, t) \in D_{e}^{-} \times \mathbb{R}\end{cases}
$$

and

$$
\mathbf{H}^{\prime}(\boldsymbol{x}, t)= \begin{cases}\mathbf{H}_{+}^{\prime}(\boldsymbol{x}, t), & (\boldsymbol{x}, t) \in D_{e}^{+} \times \mathbb{R} \\ T_{1} \mathbf{H}_{+}^{\prime}\left(T_{1} \mathbf{x}, t\right), & (\boldsymbol{x}, t) \in D_{e}^{-} \times \mathbb{R}\end{cases}
$$

Apparently $\left(\mathbf{E}^{\prime}, \mathbf{H}^{\prime}\right) \in \boldsymbol{H}_{\sigma}^{p-1}\left(\mathbb{R}_{+}, \boldsymbol{H}_{0}\left(\operatorname{curl}, D_{e}\right)\right) \times \boldsymbol{H}_{\sigma}^{p-1}\left(\mathbb{R}_{+}, \boldsymbol{H}\left(\operatorname{curl}, D_{e}\right)\right)$ satisfies equations (2.4)-(2.5). For

$$
\mathbf{n}(\boldsymbol{x}, t)=\left(\mathrm{n}_{1}(\boldsymbol{x}, t), \mathrm{n}_{2}(\boldsymbol{x}, t), \mathrm{n}_{2}(\boldsymbol{x}, t)\right)^{\mathrm{T}},
$$

the symmetric property of the boundary $\partial D$ implies

$$
\mathbf{n}(\boldsymbol{x}, t)=T_{1} \mathbf{n}\left(T_{1} \mathbf{x}, t\right) .
$$

Thus, the fact that $\mathbf{E}_{+}^{\prime}$ is the solution of (2.1)-(2.3) implies

$$
\mathbf{n} \times \mathbf{E}^{\prime}=0 \quad \text { on } \partial D \times \mathbb{R} .
$$

Then $\left(\mathbf{E}^{\prime}, \mathbf{H}^{\prime}\right)$ is also a solution of problem (2.4)-(2.6). Proposition 2.4 implies $(\mathbf{E}, \mathbf{H})=$ $\left(\mathbf{E}^{\prime}, \mathbf{H}^{\prime}\right)$. Thus

$$
\left(\mathbf{E}_{+}^{\prime}, \mathbf{H}_{+}^{\prime}\right)=\left(\mathbf{E}_{+}, \mathbf{H}_{+}\right)
$$

Then $\left(\mathbf{E}_{+}, \mathbf{H}_{+}\right)$is the unique solution of problem (2.1)-(2.3).

\section{Acknowledgements}

The authors thank the reviewers for their careful reading of the paper.

\section{Funding}

The work of Minfu Zhang and Fuming Ma was supported by the NSFC [No. 11771180]. The work of Bo Chen was supported by the NSFC [No. 11671170] and the Fundamental Research Funds for the Central Universities (Special Project for Civil Aviation University of China, No. 3122018L009). 


\section{Abbreviations}

$t$,Time; $\mathbf{x}$, Space; $\varepsilon$, Electric permittivity; $\mu$, Magnetic permeability; $\mathbf{E}$, Electric field intensity; $\mathbf{H}$, Magnetic field intensity; $\mathbf{J}$, Current density; $\mathbf{n}$, Unit outward normal surface vector; $\rho$, Charge density; $\mathbf{v}$, Particles' average drift velocity.

\section{Availability of data and materials}

Data sharing is not applicable to this article as no datasets were generated or analyzed during the current study.

\section{Competing interests}

The authors declare that they have no competing interests.

\section{Authors' contributions}

All authors contributed equally to the writing of this paper. All authors read and approved the final manuscript.

\section{Author details}

'Institute of Mathematics, Jilin University, Changchun, China. ${ }^{2}$ College of Science, Civil Aviation University of China, Tianjin, China.

\section{Publisher's Note}

Springer Nature remains neutral with regard to jurisdictional claims in published maps and institutional affiliations.

Received: 10 November 2018 Accepted: 29 January 2019 Published online: 04 February 2019

\section{References}

1. Bhatti, M.M., Lu, D.Q.: Head-on collision between two hydroelastic solitary waves in shallow water. Qual. Theory Dyn. Syst. 17, 103-122(2018)

2. Monk, P.: Finite Element Methods for Maxwell's Equations. Numerical Mathematics and Scientific Computation. Oxford University Press, New York (2003)

3. Colton, D., Kress, R.: Inverse Acoustic and Electromagnetic Scattering Theory, 3rd edn. Springer, Berlin (2013)

4. Li, J.Z., Liu, H.Y., Sun, H.P.: On a gesture-computing technique using electromagnetic waves. Inverse Probl. Imaging 12, 677-696 (2017)

5. Liu, H.Y., Wang, Y.L., Zhong, S.H.: Nearly non-scattering electromagnetic wave set and its application. Z. Angew. Math. Phys. 68, 68:35 (15 pp.) (2017)

6. Kress, R., Tran, T.: Inverse scattering for a locally perturbed half-plane. Inverse Probl. 16, 1541-1559 (2000)

7. Feng, L.X., Ma, F.M.: A sampling method for solving inverse scattering problems with a locally perturbed half plane. Northeast. Math. J. 19, 1-4 (2003)

8. Dong, H.P., Ma, F.M.: Reconstruction of the shape of object with near field measurements in a half-plane. Sci. China Math. 51, 1059-1070 (2008)

9. Dong, H.P., Ma, F.M., Yuan, X.K., Guo, Y.K.: Inverse electromagnetic scattering for a locally perturbed perfectly conducting plate. Wave Motion 60, 95-107 (2016)

10. Ammari, H., Bao, G., Wood, A.W.: An integral equation method for the electromagnetic scattering from cavities. Math. Methods Appl. Sci. 23, 1057-1072 (2015)

11. Bao, G., Yun, K.H., Zhou, Z.F.: Stability of the scattering from a large electromagnetic cavity in two dimensions. SIAM J. Math. Anal. 44, 383-404 (2012)

12. Lai, J., Greengard, L., O'Neil, M.: Robust integral formulations for electromagnetic scattering from three-dimensional cavities. J. Comput. Phys. 345, 1-16 (2017)

13. Wang, X.C., Guo, Y.K., Li, J.Z., Liu, H.Y.: Mathematical design of a novel input/instruction device using a moving emitter. Inverse Probl. 33, 105009 (19 pp.) (2017)

14. Guo, Y.K., Li, J.Z., Liu, H.Y., Wang, X.C.: Two gesture-computing approaches by using electromagnetic waves. Preprint (2017). arXiv:1705.07713v1

15. Marin, M., Öchsner, A.: The effect of a dipolar structure on the Hölder stability in Green-Naghdi thermoelasticity. Contin. Mech. Thermodyn. 29, 1365-1374 (2017)

16. Sayas, F.J.: Retarded Potentials and Time Domain Boundary Integral Equations: A Road-Map. Springer Series in Computational Mathematics, vol. 50. Springer, Bessel (2016)

17. Guo, Y.K., Hömberg, D., Hu, G.H., Li, J.Z., Liu, H.Y.: A time domain sampling method for inverse acoustic scattering problems. J. Comput. Phys. 314, 647-660 (2016)

18. Sun, Y.: Indirect boundary integral equation method for the Cauchy problem of the Laplace equation. J. Sci. Comput. 71, 469-498 (2017)

19. Laliena, A.R., Sayas, F.J.: Theoretical aspects of the application of convolution quadrature to scattering of acoustic waves. Numer. Math. 112, 637-678 (2009)

20. Chen, B., Ma, F.M., Guo, Y.K.: Time domain scattering and inverse scattering problems in a locally perturbed half-plane. Appl. Anal. 96, 1303-1325 (2017)

21. Haddar, H., Lechleiter, A., Marmorat, S.: An improved time domain linear sampling method for Robin and Neumann obstacles. Appl. Anal. 93, 369-390 (2014)

22. Chen, Q., Haddar, H., Lechleiter, A., Monk, P.: A sampling method for inverse scattering in the time domain. Inverse Probl. 26, 85001-85017 (2010)

23. Guo, Y.K., Monk, P., Colton, D.: Toward a time domain approach to the linear sampling method. Inverse Probl. 29 95016-95032(2013)

24. Gao, Y.X., Li, P.J., Zhang, B.: Analysis of transient acoustic-elastic interaction in an unbounded structure. SIAM J. Math. Anal. 49, 3951-3972 (2016)

25. Fan, L., Monk, P.: Time dependent scattering from a grating. J. Comput. Phys. 302, 97-113 (2015) 
26. Chen, Z.M., Nédélec, J.C.: On Maxwell equations with the transparent boundary condition. J. Comput. Math. 26 284-296 (2008)

27. Gao, Y.X., Li, P.J.: Analysis of time-domain scattering by periodic structures. J. Differ. Equ. 261, 5094-5118 (2016)

28. Gao, Y.X., Li, P.J.: Electromagnetic scattering for time-domain Maxwell's equations in an unbounded structure. Math. Models Methods Appl. Sci. 27, 1843-1870 (2017)

29. Li, P.J.: A survey of open cavity scattering problems. J. Comput. Math. 36, 1-16 (2018)

30. Li, P.J., Wu, H.J., Zheng, W.Y.: An overfilled cavity problem for Maxwell's equations. J. Differ. Equ. 252, 3209-3225 (2012)

31. Li, P.J., Wang, L.L., Wood, A.: Analysis of transient electromagnetic scattering from a three-dimensional open cavity. SIAM J. Appl. Math. 75, 1675-1699 (2015)

32. Wood, A.: Analysis of electromagnetic scattering from an overfilled cavity in the ground plane. J. Comput. Phys. 215 630-641 (2006)

33. Kravchenko, V.V., Oviedo, H.: Time-dependent electromagnetic fields in chiral media. J. Phys. A, Math. Theor. 43, 455213 (9 pp.) (2010)

34. Liu, H.Y., Rondi, L., Xiao, J.N.: Mosco convergence for H(curl) spaces, higher integrability for Maxwell's equations, and stability in direct and inverse EM scattering problems. Preprint, (2016). arXiv:1603.07555

\section{Submit your manuscript to a SpringerOpen ${ }^{\circ}$} journal and benefit from:

- Convenient online submission

- Rigorous peer review

- Open access: articles freely available online

- High visibility within the field

- Retaining the copyright to your article

Submit your next manuscript at $\gg$ springeropen.com 